\title{
In situ TEM Study of Confined Dislocation Nucleation Processes
}

\author{
M. Legros, F. Mompiou*, D. S. Gianola**, A. Stedlmayr***, and D. Caillard* \\ * CEMES-CNRS, 29 rue Jeanne Marvig, Toulouse, France \\ ** University of Pennsylvania, Dept of Mater. Sci and Eng., Philadelphia, USA \\ *** Karlsruhe Institute für Technologie (KIT) Institut für Materialforschung, 76344 Eggenstein- \\ Leopoldshafen, Germany
}

The strength of metals and alloys depends intrinsically on the ability of their dislocations to multiply and travel over large distances inside the crystalline lattice. When reduced to very small dimensions such as in thin films, whiskers or micro-pillars, dislocations can escape more easily through free surfaces before interacting with others. For this reason, these small crystals do not harden when strained. Their initial resistance to deformation (yield strength) is however much larger than their bulk counterpart, and the physical explanations for this outstanding resistance are still highly debated

One critical contribution lies in the nucleation of fresh dislocations. The classical source, represented by the Franck-Read scheme, is unlikely to operate in sub-micron size crystals despite its well spread use in computer-based models. Overall, the nucleation process in small structures is much less documented than dislocation-dislocation interactions, and this limited knowledge partly lies in the stochastic nature of sources and the difficulty to observe them while operating.

In the present work, we relate an extensive in situ transmission electron microscope (TEM) study of dislocation multiplication and shearing processes in sub-micrometre Al ligaments (Fig. 1), fibers (Fig. 2), and films that were kept free of FIB (Focused Ion Beam) during preparation. Operating sources, often single armed (Fig. 1), and other nucleation modes (open loops produced by double cross-slip, Fig. 2.) have been monitored in real time, analyzed and compared to the crystal dimensions. The stress measurements withdrawn from mobile dislocation curvatures will be compared to micro-tensile tests. Possible strengthening mechanisms will be discussed in the light of these results and confronted with recent results obtained in similar experiments [1] and simulations [2]. These experiments clearly underline the prominent role of dislocation nucleation in setting the stress resistance of small crystals.

\section{References}

[1] D. Kiener, et al., Acta Materialia, 56 (2008), 580.

[2] C. Motz, et al., Acta Materialia, 57 (2009), 1744-1754.

[3] S.H. Oh, et al., Nature Materials, 8 (2009), 95-100. 


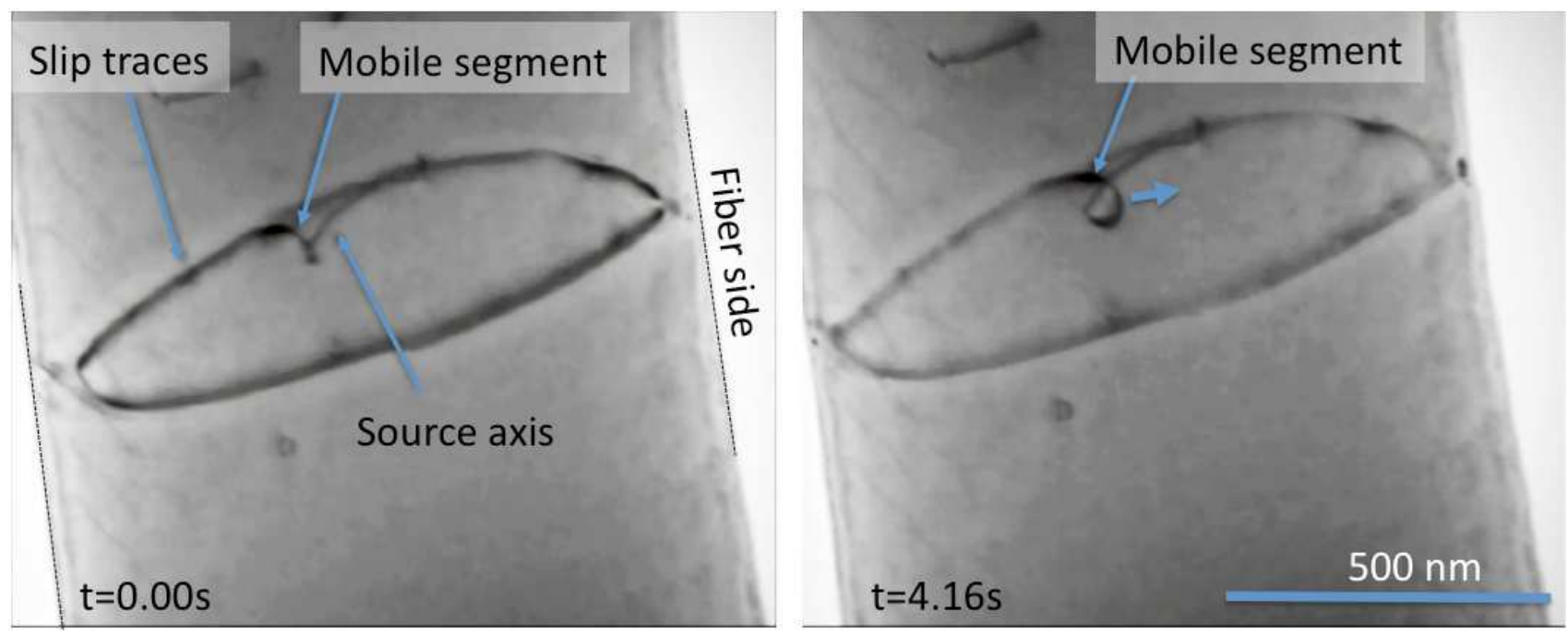

FIG. 1. Single arm dislocation source activated in an Al fiber during an in situ TEM straining experiment. Straining axis is vertical. Slip traces, that correspond to the intersection of the slip plane and the surface indicate, in projection, the shape of the fiber.

\section{Fresh dislocation segment}

\section{Open loop}

\section{Polyimide}

\section{$200 \mathrm{~nm}$}

FIG. 2. Dislocation multiplication by double cross-slip process in a $400 \mathrm{~nm}$ wide Al single crystal ligament. During the sample preparation, the metal was protected from the FIB irradiation by the polyimide substrate that broke during stretching, letting bare the Al crystal (after [3]). Straining axis is vertical. 